\title{
Precision Measurement of Neutrino Oscillation Parameters with KamLAND
}

S. Abe, ${ }^{1}$ T. Ebihara, ${ }^{1}$ S. Enomoto, ${ }^{1}$ K. Furuno, ${ }^{1}$ Y. Gando, ${ }^{1}$ K. Ichimura, ${ }^{1}$ H. Ikeda, ${ }^{1}$ K. Inoue, ${ }^{1}$ Y. Kibe, ${ }^{1}$ Y. Kishimoto, ${ }^{1}$ M. Koga,${ }^{1}$ A. Kozlov, ${ }^{1}$ Y. Minekawa, ${ }^{1}$ T. Mitsui, ${ }^{1}$ K. Nakajima, ${ }^{1, *}$ K. Nakajima, ${ }^{1}$ K. Nakamura, ${ }^{1}$ M. Nakamura, ${ }^{1}$ K. Owada, ${ }^{1}$ I. Shimizu, ${ }^{1}$ Y. Shimizu, ${ }^{1}$ J. Shirai, ${ }^{1}$ F. Suekane, ${ }^{1}$ A. Suzuki, ${ }^{1}$ Y. Takemoto, ${ }^{1}$ K. Tamae, ${ }^{1}$ A. Terashima, ${ }^{1}$ H. Watanabe, ${ }^{1}$ E. Yonezawa, ${ }^{1}$ S. Yoshida, ${ }^{1}$ J. Busenitz, ${ }^{2}$ T. Classen, ${ }^{2}$ C. Grant,${ }^{2}$ G. Keefer, ${ }^{2}$ D. S. Leonard,${ }^{2}$ D. McKee, ${ }^{2}$ A. Piepke, ${ }^{2}$ M. P. Decowski, ${ }^{3}$ J. A. Detwiler, ${ }^{3}$ S. J. Freedman, ${ }^{3}$ B. K. Fujikawa, ${ }^{3}$ F. Gray, ${ }^{3, \dagger}$ E. Guardincerri, ${ }^{3}$ L. Hsu, ${ }^{3, \neq}$ R. Kadel, ${ }^{3}$ C. Lendvai, ${ }^{3}$ K.-B. Luk, ${ }^{3}$ H. Murayama, ${ }^{3}$ T. O'Donnell, ${ }^{3}$ H. M. Steiner, ${ }^{3}$ L. A. Winslow, ${ }^{3}$ D. A. Dwyer, ${ }^{4}$ C. Jillings, ${ }^{4,}$ C. Mauger, ${ }^{4}$ R. D. McKeown,${ }^{4}$ P. Vogel,${ }^{4}$ C. Zhang, ${ }^{4}$ B. E. Berger, ${ }^{5}$ C. E. Lane, ${ }^{6}$ J. Maricic, ${ }^{6}$ T. Miletic, ${ }^{6}$ M. Batygov, ${ }^{7}$ J. G. Learned, ${ }^{7}$ S. Matsuno, ${ }^{7}$ S. Pakvasa, ${ }^{7}$ J. Foster,${ }^{8}$ G. A. Horton-Smith, ${ }^{9}$ A. Tang, ${ }^{8}$ S. Dazeley, ${ }^{9}, \|$ K. E. Downum, ${ }^{10}$ G. Gratta, ${ }^{10}$ K. Tolich,${ }^{10}$ W. Bugg, ${ }^{11}$ Y. Efremenko, ${ }^{11}$ Y. Kamyshkov,${ }^{11}$ O. Perevozchikov, ${ }^{11}$ H. J. Karwowski, ${ }^{12}$ D. M. Markoff, ${ }^{12}$ W. Tornow, ${ }^{12}$ K. M. Heeger, ${ }^{13}$ F. Piquemal, ${ }^{14}$ and J.-S. Ricol ${ }^{14}$

(The KamLAND Collaboration)

\author{
${ }^{1}$ Research Center for Neutrino Science, Tohoku University, Sendai 980-8578, Japan \\ ${ }^{2}$ Department of Physics and Astronomy, University of Alabama, Tuscaloosa, Alabama 35487, USA \\ ${ }^{3}$ Physics Department, University of California, Berkeley and Lawrence Berkeley National Laboratory, \\ Berkeley, California 94720, USA \\ ${ }^{4}$ W. K. Kellogg Radiation Laboratory, California Institute of Technology, Pasadena, California 91125, USA \\ ${ }^{5}$ Department of Physics, Colorado State University, Fort Collins, Colorado 80523, USA \\ ${ }^{6}$ Physics Department, Drexel University, Philadelphia, Pennsylvania 19104, USA \\ ${ }^{7}$ Department of Physics and Astronomy, University of Hawaii at Manoa, Honolulu, Hawaii 96822, USA \\ ${ }^{8}$ Department of Physics, Kansas State University, Manhattan, Kansas 66506, USA \\ ${ }^{9}$ Department of Physics and Astronomy, Louisiana State University, Baton Rouge, Louisiana 70803, USA \\ ${ }^{10}$ Physics Department, Stanford University, Stanford, California 94305, USA \\ ${ }^{11}$ Department of Physics and Astronomy, University of Tennessee, Knoxville, Tennessee 37996, USA \\ ${ }^{12}$ Triangle Universities Nuclear Laboratory, Durham, North Carolina 27708, USA \\ and Physics Departments at Duke University, North Carolina Central University, \\ and the University of North Carolina at Chapel Hill, North Carolina, USA \\ ${ }^{13}$ Department of Physics, University of Wisconsin, Madison, Wisconsin 53706, USA \\ ${ }^{14}$ CEN Bordeaux-Gradignan, IN2P3-CNRS and University Bordeaux I, F-33175 Gradignan Cedex, France
}

(Received 29 January 2008; published 5 June 2008)

\begin{abstract}
The KamLAND experiment has determined a precise value for the neutrino oscillation parameter $\Delta m_{21}^{2}$ and stringent constraints on $\theta_{12}$. The exposure to nuclear reactor antineutrinos is increased almost fourfold over previous results to $2.44 \times 10^{32}$ proton yr due to longer livetime and an enlarged fiducial volume. An undistorted reactor $\bar{\nu}_{e}$ energy spectrum is now rejected at $>5 \sigma$. Analysis of the reactor spectrum above the inverse beta decay energy threshold, and including geoneutrinos, gives a best fit at $\Delta m_{21}^{2}=$ $7.58_{-0.13}^{+0.14}(\text { stat })_{-0.15}^{+0.15}$ (syst) $\times 10^{-5} \mathrm{eV}^{2}$ and $\tan ^{2} \theta_{12}=0.56_{-0.07}^{+0.10}(\text { stat })_{-0.06}^{+0.10}($ syst $)$. Local $\Delta \chi^{2}$ minima at higher and lower $\Delta m_{21}^{2}$ are disfavored at $>4 \sigma$. Combining with solar neutrino data, we obtain $\Delta m_{21}^{2}=$ $7.59_{-0.21}^{+0.21} \times 10^{-5} \mathrm{eV}^{2}$ and $\tan ^{2} \theta_{12}=0.47_{-0.05}^{+0.06}$.

DOI: 10.1103/PhysRevLett.100.221803

PACS numbers: 14.60.Pq, 26.65.+t, 28.50.Hw, 91.35.-x
\end{abstract}

Experiments studying atmospheric, solar, reactor, and accelerator neutrinos provide compelling evidence for neutrino mass and oscillation. The Kamioka Liquid scintillator Anti-Neutrino Detector (KamLAND) investigates neutrino oscillation parameters by observing electron antineutrinos $\left(\bar{\nu}_{e}\right)$ emitted from distant nuclear reactors. Previously, KamLAND announced the first evidence of $\bar{\nu}_{e}$ disappearance [1], followed by direct evidence for neutrino oscillation by observing distortion of the reactor $\bar{\nu}_{e}$ energy spectrum [2]. More recently, KamLAND showed the first indication of geologically produced antineutrinos (geoneu- trinos) from radioactive decay in the Earth [3], possibly a unique tool for geology.

This Letter presents a precise measurement of $\Delta m_{21}^{2}$ and new constraints on $\theta_{12}$ based on data collected from March 9, 2002 to May 12, 2007, including data used earlier [1,2]. We have enlarged the fiducial volume radius from 5.5 to $6 \mathrm{~m}$ and collected significantly more data; the total exposure is $2.44 \times 10^{32}$ proton yr ( 2881 ton $\mathrm{yr}$ ). We have expanded the analysis to the full reactor $\bar{\nu}_{e}$ energy spectrum and reduced the systematic uncertainties in the number of target protons and the background. We now observe almost 
two complete oscillation cycles in the $\bar{\nu}_{e}$ spectrum and extract more precise values of the oscillation parameters.

KamLAND is at the site of the former Kamiokande experiment at a depth of $\sim 2700 \mathrm{~m}$ water equivalent. The heart of the detector is 1 kton of highly purified liquid scintillator (LS) enclosed in an EVOH/nylon balloon suspended in purified mineral oil. The LS consists of $80 \%$ dodecane, $20 \%$ pseudocumene, and $1.36 \pm 0.03 \mathrm{~g} / 1$ of PPO [4]. The antineutrino detector is inside an $18-\mathrm{m}-$ diameter stainless steel sphere. An array of $187950-\mathrm{cm}-$ diameter photomultiplier tubes (PMTs) is mounted on the inner surface of the sphere. A subset of 554 PMTs are reused from the Kamiokande experiment, while the remaining 1325 PMTs are a faster version masked to 17 inches. A 3.2-kton cylindrical water-Cherenkov outer detector (OD), surrounding the containment sphere, provides shielding and operates as an active cosmic-ray veto detector.

Electron antineutrinos are detected via inverse $\beta$-decay, $\bar{\nu}_{e}+p \rightarrow e^{+}+n$, with a $1.8 \mathrm{MeV}$ threshold. The prompt scintillation light from the $e^{+}$gives a measure of the $\bar{\nu}_{e}$ energy, $E_{\bar{\nu}_{e}} \simeq E_{p}+\bar{E}_{n}+0.8 \mathrm{MeV}$, where $E_{p}$ is the prompt event energy including the positron kinetic and annihilation energy, and $\bar{E}_{n}$ is the average neutron recoil energy, $O(10 \mathrm{keV})$. The mean neutron capture time is $207.5 \pm 2.8 \mu \mathrm{s}$. More than $99 \%$ capture on free protons, producing a $2.2 \mathrm{MeV} \gamma$ ray.

KamLAND is surrounded by 55 Japanese nuclear power reactor units, each an isotropic $\bar{\nu}_{e}$ source. The reactor operation records, including thermal power generation, fuel burnup, and exchange and enrichment logs, are provided by a consortium of Japanese electric power companies. This information, combined with publicly available world reactor data, is used to calculate the instantaneous fission rates using a reactor model [5]. Only four isotopes contribute significantly to the $\bar{\nu}_{e}$ spectra; the ratios of the fission yields averaged over the entire data taking period are: $\quad{ }^{235} \mathrm{U}:{ }^{238} \mathrm{U}:{ }^{239} \mathrm{Pu}:{ }^{241} \mathrm{Pu}=0.570: 0.078: 0.295: 0.057$. The emitted $\bar{\nu}_{e}$ energy spectrum is calculated using the $\bar{\nu}_{e}$ spectra inferred from Ref. [6], while the spectral uncertainty is evaluated from Ref. [7]. We also include contributions from the long-lived fission daughters ${ }^{90} \mathrm{Sr},{ }^{106} \mathrm{Ru}$, and ${ }^{144} \mathrm{Ce}[8]$.

We recently commissioned an "off-axis" calibration system capable of positioning radioactive sources away from the central vertical axis of the detector. The measurements indicate that the vertex reconstruction systematic deviations are radius- and zenith-angle-dependent, but smaller than $3 \mathrm{~cm}$ and independent of azimuthal angle. The fiducial volume (FV) is known to $1.6 \%$ uncertainty up to $5.5 \mathrm{~m}$ using the off-axis calibration system. The position distribution of the $\beta$-decays of muon-induced ${ }^{12} \mathrm{~B} /{ }^{12} \mathrm{~N}$ confirms this with $4.0 \%$ uncertainty by comparing the number of events inside $5.5 \mathrm{~m}$ to the number produced in the full LS volume. The ${ }^{12} \mathrm{~B} /{ }^{12} \mathrm{~N}$ event ratio is used to establish the uncertainty between 5.5 and $6 \mathrm{~m}$, resulting in a combined 6-m-radius FV uncertainty of $1.8 \%$.
Off-axis calibration measurements and numerous central-axis deployments of ${ }^{60} \mathrm{Co},{ }^{68} \mathrm{Ge},{ }^{203} \mathrm{Hg},{ }^{65} \mathrm{Zn}$, ${ }^{241} \mathrm{Am}^{9} \mathrm{Be},{ }^{137} \mathrm{Cs}$, and ${ }^{210} \mathrm{Po}^{13} \mathrm{C}$ radioactive sources established the event reconstruction performance. The vertex reconstruction resolution is $\sim 12 \mathrm{~cm} / \sqrt{E(\mathrm{MeV})}$, and the energy resolution is $6.5 \% / \sqrt{E(\mathrm{MeV})}$. The scintillator response is corrected for the nonlinear effects from quenching and Cherenkov light production. The systematic variation of the energy reconstruction over the data set give an absolute energy-scale uncertainty of $1.4 \%$; the distortion of the energy scale results in a $1.9 \%$ uncertainty on $\Delta m_{21}^{2}$, while the uncertainty at the analysis threshold gives a $1.5 \%$ uncertainty on the event rate. Table I summarizes the systematic uncertainties. The total uncertainty on $\Delta m_{21}^{2}$ is $2.0 \%$, while the uncertainty on the expected event rate, which primarily affects $\theta_{12}$, is $4.1 \%$.

For the analysis, we require $0.9 \mathrm{MeV}<E_{p}<8.5 \mathrm{MeV}$. The delayed energy, $E_{d}$, must satisfy $1.8 \mathrm{MeV}<E_{d}<$ $2.6 \mathrm{MeV}$ or $4.0 \mathrm{MeV}<E_{d}<5.8 \mathrm{MeV}$, corresponding to the neutron-capture $\gamma$ energies for $p$ and ${ }^{12} \mathrm{C}$, respectively. The time difference $(\Delta T)$ and distance $(\Delta R)$ between the prompt event and delayed neutron capture are selected to be $0.5 \mu \mathrm{s}<\Delta T<1000 \mu \mathrm{s}$ and $\Delta R<2 \mathrm{~m}$. The prompt and delayed radial distance from the detector center $\left(R_{p}\right.$, $R_{d}$ ) must be $<6 \mathrm{~m}$.

Accidental coincidences increase near the balloon surface $(R=6.5 \mathrm{~m})$, reducing the signal-to-background ratio. We use constraints on event characteristics to suppress accidental backgrounds while maintaining high efficiency. We construct a probability density function (PDF) for accidental coincidence events, $f_{a c c}\left(E_{p}, E_{d}, \Delta R, \Delta T, R_{p}, R_{d}\right)$, by pairing events in a $10-$ to 20-s delayed-coincidence window. A PDF for the $\bar{\nu}_{e}$ signal, $f_{\bar{\nu}_{e}}\left(E_{p}, E_{d}, \Delta R, \Delta T, R_{p}, R_{d}\right)$, is constructed from a Monte Carlo simulation of the prompt and delayed events using the measured neutron capture time and detector response. For the $E_{p}$ distribution in $f_{\bar{\nu}_{e}}$, we choose an oscillation-free reactor spectrum including a contribution from geoneutrinos estimated from Ref. [9]. A discriminator value, $L=\frac{f_{\bar{\nu}_{e}}}{f_{\bar{\nu}_{e}}+f_{a c c}}$, is calculated for each candidate pair that passes the earlier cuts. We establish a selection value $L_{i}^{\text {cut }}$ in $E_{p}$ bins of $0.1 \mathrm{MeV}$, where $L_{i}^{\text {cut }}$ is the value of $L$ at which the figure-of-merit, $\frac{S_{i}}{\sqrt{S_{i}+B_{i}}}$ is maximal. $S_{i}$ is the

TABLE I. Estimated systematic uncertainties relevant for the neutrino oscillation parameters $\Delta m_{21}^{2}$ and $\theta_{12}$.

\begin{tabular}{lllll}
\hline \hline & \multicolumn{2}{l}{ Detector-related $(\%)$} & \multicolumn{2}{c}{ Reactor-related (\%) } \\
\hline$\Delta m_{21}^{2}$ & Energy scale & 1.9 & $\bar{\nu}_{e}$-spectra [7] & 0.6 \\
Event rate & Fiducial volume & 1.8 & $\bar{\nu}_{e}$-spectra & 2.4 \\
& Energy threshold & 1.5 & Reactor power & 2.1 \\
& Efficiency & 0.6 & Fuel composition & 1.0 \\
& Cross section & 0.2 & Long-lived nuclei & 0.3 \\
\hline \hline
\end{tabular}




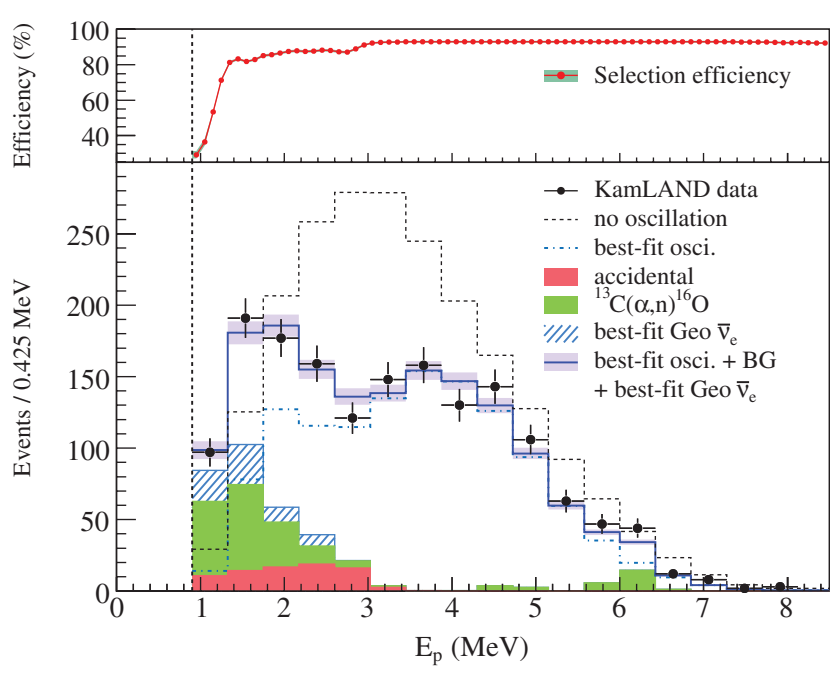

FIG. 1 (color). Prompt event energy spectrum of $\bar{\nu}_{e}$ candidate events. All histograms corresponding to reactor spectra and expected backgrounds incorporate the energy-dependent selection efficiency (top panel). The shaded background and geoneutrino histograms are cumulative. Statistical uncertainties are shown for the data; the band on the blue histogram indicates the event rate systematic uncertainty.

number of Monte Carlo signal events in the $i$ th energy bin with $L>L_{i}^{\text {cut }} . B_{i}$ is calculated similarly using the accidental coincidence event pairs. The choice of the $E_{p}$ distribution in $f_{\bar{\nu}_{e}}$ affects only the discrimination power of the procedure; substituting the oscillation-free reactor spectrum by an oscillated spectrum with the parameters from Ref. [2] changes our oscillation parameter results by less than $0.2 \sigma$. The selection efficiency $\epsilon\left(E_{p}\right)$ is estimated from the fraction of selected coincidence events relative to the total generated in $R<6 \mathrm{~m}$ in the simulation, see Fig. 1 (top).

The dominant background is caused by ${ }^{13} \mathrm{C}(\alpha, n){ }^{16} \mathrm{O}$ reactions from $\alpha$-decay of ${ }^{210} \mathrm{Po}$, a daughter of ${ }^{222} \mathrm{Rn}$ introduced into the LS during construction. We estimate that there are $(5.56 \pm 0.22) \times 10^{9}{ }^{210} \mathrm{Po} \alpha$-decays. The ${ }^{13} \mathrm{C}(\alpha, n){ }^{16} \mathrm{C}$ reaction results in neutrons with energies up to $7.3 \mathrm{MeV}$, but most of the scintillation energy spectrum is quenched below $2.7 \mathrm{MeV}$. In addition, ${ }^{12} \mathrm{C}\left(n, n^{\prime}\right)^{12} \mathrm{C}^{*}$, and the 1st and 2 nd excited states of ${ }^{16} \mathrm{O}$ produce signals in coincidence with the scattered neutron but the cross sections are not known precisely. $\mathrm{A}{ }^{210} \mathrm{Po}^{13} \mathrm{C}$ source was employed to study the ${ }^{13} \mathrm{C}(\alpha, n){ }^{16} \mathrm{C}$ reaction and tune a simulation using the cross sections from Refs. [10,11]. We find that the cross sections for the excited ${ }^{16} \mathrm{O}$ states from Ref. [10] agree with the ${ }^{210} \mathrm{Po}^{13} \mathrm{C}$ data after scaling the 1 st excited state by 0.6 ; the 2 nd excited state requires no scaling. For the ground state, we use the cross section from Ref. [11] and scale by 1.05 . Including the ${ }^{210} \mathrm{Po}$ decay-rate, we assign an uncertainty of $11 \%$ for the ground state and $20 \%$ for the excited states. Accounting for $\epsilon\left(E_{p}\right)$,
TABLE II. Estimated backgrounds after selection efficiencies.

\begin{tabular}{lc}
\hline \hline \multicolumn{1}{c}{ Background } & Contribution \\
\hline Accidentals & $80.5 \pm 0.1$ \\
${ }^{9} \mathrm{Li} /{ }^{8} \mathrm{He}$ & $13.6 \pm 1.0$ \\
Fast neutron \& Atmospheric $\nu$ & $<9.0$ \\
${ }^{13} \mathrm{C}(\alpha, n){ }^{16} \mathrm{O}_{g s}, n p \rightarrow n p$ & $157.2 \pm 17.3$ \\
${ }^{13} \mathrm{C}(\alpha, n){ }^{16} \mathrm{O}_{g s},{ }^{12} \mathrm{C}\left(n, n{ }^{\prime}\right){ }^{12} \mathrm{C}^{*}(4.4 \mathrm{MeV} \gamma)$ & $6.1 \pm 0.7$ \\
${ }^{13} \mathrm{C}(\alpha, n){ }^{16} \mathrm{O} 1$ st exc. state $\left(6.05 \mathrm{MeV} e e^{+}\right)$ & $15.2 \pm 3.5$ \\
${ }^{13} \mathrm{C}(\alpha, n){ }^{16} \mathrm{O}$ 2nd exc. state $(6.13 \mathrm{MeV} \gamma)$ & $3.5 \pm 0.2$ \\
Total & $276.1 \pm 23.5$ \\
\hline \hline
\end{tabular}

there should be $182.0 \pm 21.7{ }^{13} \mathrm{C}(\alpha, n){ }^{16} \mathrm{O}$ events in the data.

To mitigate background arising from the cosmogenic beta delayed-neutron emitters ${ }^{9} \mathrm{Li}$ and ${ }^{8} \mathrm{Li}$, we apply a $2 \mathrm{~s}$ veto within a 3-m-radius cylinder around well-identified muon tracks passing through the LS. For muons that either deposit a large amount of energy or cannot be tracked, we apply a $2 \mathrm{~s}$ veto of the full detector. We estimate that $13.6 \pm 1.0$ events from ${ }^{9} \mathrm{Li} /{ }^{8} \mathrm{He}$ decays remain by fitting the time distribution of identified ${ }^{9} \mathrm{Li} /{ }^{8} \mathrm{He}$ since the prior muons. Spallation-produced neutrons are suppressed with a $2 \mathrm{~ms}$ full-volume veto after a detected muon. Some neutrons are produced by muons that are undetected by the OD or miss the OD but interact in the nearby rock. These neutrons can scatter and capture in the LS, mimicking the $\bar{\nu}_{e}$ signal. We also expect background events from atmospheric neutrinos. The energy spectrum of these back-

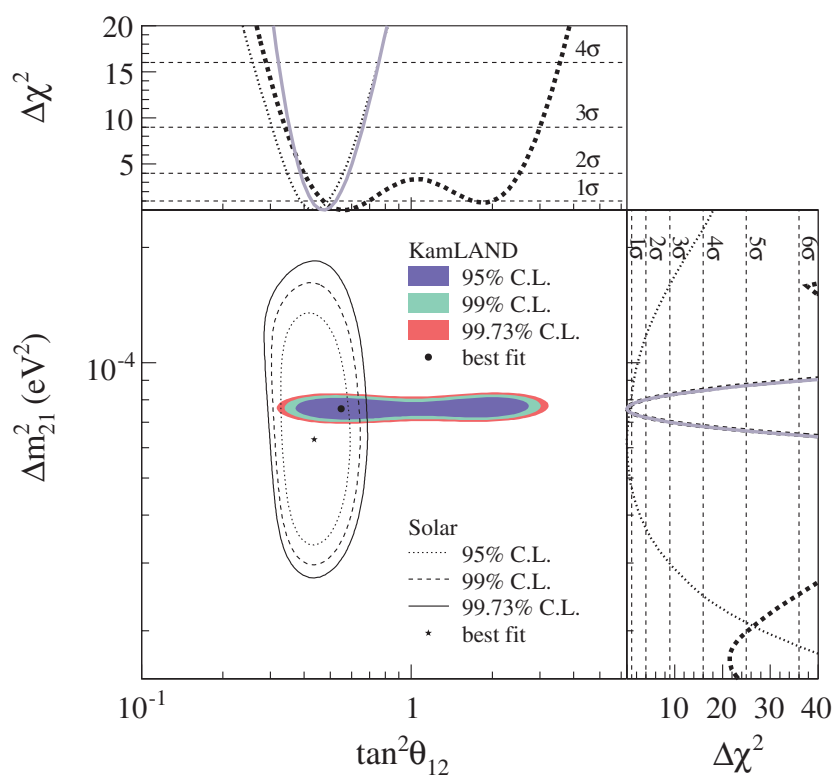

FIG. 2 (color). Allowed region for neutrino oscillation parameters from KamLAND and solar neutrino experiments. The side-panels show the $\Delta \chi^{2}$-profiles for KamLAND (dashed line) and solar experiments (dotted line) individually, as well as the combination of the two (solid line). 


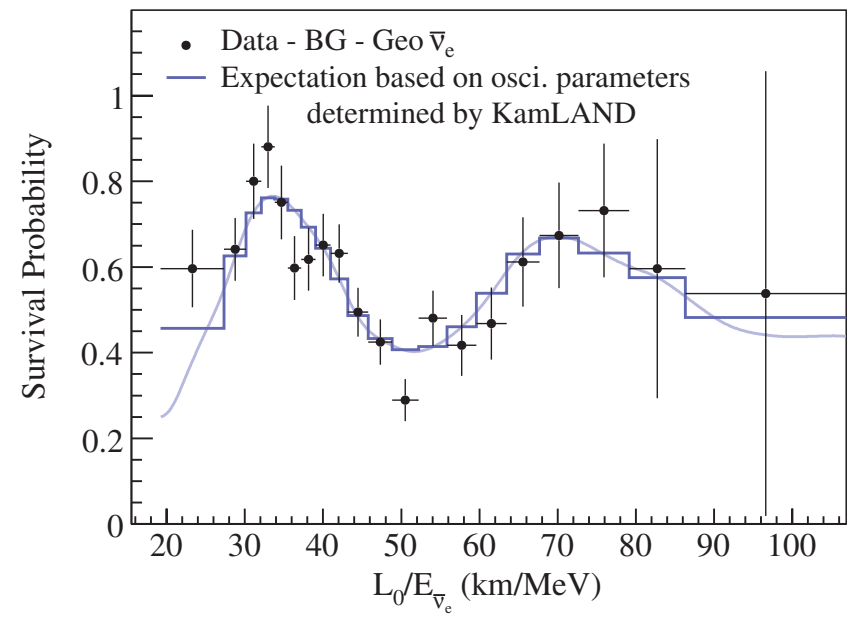

FIG. 3 (color). Ratio of the background and geoneutrinosubtracted $\bar{\nu}_{e}$ spectrum to the expectation for no-oscillation as a function of $L_{0} / E$. $L_{0}$ is the effective baseline taken as a fluxweighted average $\left(L_{0}=180 \mathrm{~km}\right)$. The energy bins are equal probability bins of the best fit including all backgrounds (see Fig. 1). The histogram and curve show the expectation accounting for the distances to the individual reactors, time-dependent flux variations, and efficiencies. The error bars are statistical only and do not include, for example, correlated systematic uncertainties in the energy scale.

grounds is assumed to be flat to at least $30 \mathrm{MeV}$ based on a simulation following [12]. The atmospheric $\nu$ spectrum [13] and interactions were modeled using NUANCE [14]. We expect fewer than 9 neutron and atmospheric $\nu$ events in the data-set. We observe 15 events in the energy range 8.5-30 MeV, consistent with the limit reported previously [15].

The accidental coincidence background above $0.9 \mathrm{MeV}$ is measured with a 10 - to 20 -s delayed-coincidence window to be $80.5 \pm 0.1$ events. Other backgrounds from ( $\gamma$, $n$ ) interactions and spontaneous fission are negligible.

Antineutrinos produced in the decay chains of ${ }^{232} \mathrm{Th}$ and ${ }^{238} \mathrm{U}$ in the Earth's interior are limited to prompt energies below $2.6 \mathrm{MeV}$. The expected geoneutrino flux at the KamLAND location is estimated with a geological reference model [9], which assumes a radiogenic heat production rate of $16 \mathrm{TW}$ from the $\mathrm{U}$ and Th-decay chains. The calculated $\bar{\nu}_{e}$ fluxes for $\mathrm{U}$ and Th-decay, including a suppression factor of 0.57 due to neutrino oscillation, are $2.24 \times 10^{6} \mathrm{~cm}^{-2} \mathrm{~s}^{-1}$ (56.6 events) and $1.90 \times$ $10^{6} \mathrm{~cm}^{-2} \mathrm{~s}^{-1}$ (13.1 events), respectively.

With no $\bar{\nu}_{e}$ disappearance, we expect $2179 \pm 89$ (syst) events from reactors. The backgrounds in the reactor energy region listed in Table II sum to $276.1 \pm 23.5$; we also expect geoneutrinos. We observe 1609 events.

Figure 1 shows the prompt energy spectrum of selected $\bar{\nu}_{e}$ events and the fitted backgrounds. The unbinned data are assessed with a maximum likelihood fit to two-flavor neutrino oscillation (with $\theta_{13}=0$ ), simultaneously fitting the geoneutrino contribution. The method incorporates the absolute time of the event and accounts for time variations in the reactor flux. Earth-matter oscillation effects are included. The best fit is shown in Fig. 1. The joint confidence intervals give $\Delta m_{21}^{2}=7.58_{-0.13}^{+0.14}(\text { stat })_{-0.15}^{+0.15}$ (syst $) \times$ $10^{-5} \mathrm{eV}^{2}$ and $\tan ^{2} \theta_{12}=0.56_{-0.07}^{+0.10}(\mathrm{stat})_{-0.06}^{+0.10}$ (syst) for $\tan ^{2} \theta_{12}<1$. A scaled reactor spectrum with no distortion from neutrino oscillation is excluded at more than $5 \sigma$. An independent analysis using cuts similar to Ref. [2] gives $\Delta m_{21}^{2}=7.66_{-0.20}^{+0.22} \times 10^{-5} \mathrm{eV}^{2}$ and $\tan ^{2} \theta_{12}=0.52_{-0.10}^{+0.16}$

The allowed contours in the neutrino oscillation parameter space, including $\Delta \chi^{2}$-profiles, are shown in Fig. 2. Only the so-called LMA-I region remains, while other regions previously allowed by KamLAND at $\sim 2.2 \sigma$ are disfavored at more than $4 \sigma$. For three-neutrino oscillation, the data give the same result for $\Delta m_{21}^{2}$, but a slightly larger uncertainty on $\theta_{12}$. Incorporating the results of SNO [16] and solar flux experiments [17] in a two-neutrino analysis with KamLAND assuming $C P T$ invariance, gives $\Delta m_{21}^{2}=$ $7.59_{-0.21}^{+0.21} \times 10^{-5} \mathrm{eV}^{2}$ and $\tan ^{2} \theta_{12}=0.47_{-0.05}^{+0.06}$.

To determine the number of geoneutrinos, we fit the normalization of the $\bar{\nu}_{e}$ energy spectrum from the $\mathrm{U}$ and Th-decay chains simultaneously with the neutrino oscillation parameters using the KamLAND and solar data. There is a strong anticorrelation between the $\mathrm{U}$ and Th-decay chain geoneutrinos, and an unconstrained fit of the individual contributions does not give meaningful results. Fixing the $\mathrm{Th} / \mathrm{U}$ mass ratio to 3.9 from planetary data [18], we obtain a combined $U+$ Th best fit value of (4.4 \pm 1.6) $\times 10^{6} \mathrm{~cm}^{-2} \mathrm{~s}^{-1}(73 \pm 27$ events), in agreement with the reference model.

The KamLAND data, together with the solar $\nu$ data, set an upper limit of $6.2 \mathrm{TW}$ (90\% C.L.) for a $\bar{\nu}_{e}$ reactor source at the Earth's center [19], assuming that the reactor produces a spectrum identical to that of a slow neutron artificial reactor.

The ratio of the background-subtracted $\bar{\nu}_{e}$ candidate events, including the subtraction of geoneutrinos, to nooscillation expectation is plotted in Fig. 3 as a function of $L_{0} / E$. The spectrum indicates almost two cycles of the periodic feature expected from neutrino oscillation.

In conclusion, KamLAND confirms neutrino oscillation, providing the most precise value of $\Delta m_{21}^{2}$ to date and improving the precision of $\tan ^{2} \theta_{12}$ in combination with solar $\nu$ data. The indication of an excess of low-energy antineutrinos consistent with an interpretation as geoneutrinos persists.

The KamLAND experiment is supported by the Japanese Ministry of Education, Culture, Sports, Science and Technology, and under the United States Department of Energy Office Grant No. DEFG03-00ER41138 and other DOE grants to individual institutions. The reactor data are provided by courtesy of the following electric associations in Japan: Hokkaido, Tohoku, Tokyo, Hokuriku, Chubu, Kansai, Chugoku, Shikoku, and 
Kyushu Electric Power Companies, Japan Atomic Power Co., and Japan Nuclear Cycle Development Institute. The Kamioka Mining and Smelting Company has provided service for activities in the mine.

*Present address: Center of Quantum Universe, Okayama University, Okayama 700-8530, Japan.

†Present address: Regis University, Denver, CO 80221, USA.

${ }^{\ddagger}$ Present address: FNAL, Batavia, IL 60510, USA. ${ }^{\S}$ Present address: SNOLAB, Lively, ON P3Y 1M3, Canada. "Present address: LLNL, Livermore, CA 94550, USA.

[1] K. Eguchi et al. (KamLAND), Phys. Rev. Lett. 90, 021802 (2003).

[2] T. Araki et al. (KamLAND), Phys. Rev. Lett. 94, 081801 (2005).

[3] T. Araki et al. (KamLAND), Nature (London) 436, 499 (2005).

[4] Previous publications incorrectly indicated $1.52 \mathrm{~g} / 1$ of PPO.

[5] K. Nakajima et al., Nucl. Instrum. Methods Phys. Res., Sect. A 569, 837 (2006).
[6] ${ }^{235}$ U: K. Schreckenbach et al., Phys. Lett. B 160, 325 (1985); ${ }^{239,241}$ Pu: A. A. Hahn et al., Phys. Lett. B 218, 365 (1989); ${ }^{238}$ U: P. Vogel et al., Phys. Rev. C 24, 1543 (1981).

[7] B. Achkar et al., Phys. Lett. B 374, 243 (1996).

[8] V. I. Kopeikin, L. A. Mikaelyan, and V. V. Sinev, Yad. Fiz. 64, 914 (2001) [Phys. At. Nucl. 64, 849 (2001)].

[9] S. Enomoto et al., Earth Planet. Sci. Lett. 258, 147 (2007).

[10] JENDL, the Japanese Evaluated Nuclear Data Library available at http://wwwndc.tokai-sc.jaea.go.jp/jendl/ jendl.html (2005)

[11] S. Harissopulos et al., Phys. Rev. C 72, 062801 (2005).

[12] M. G. Marino et al., Nucl. Instrum. Methods Phys. Res., Sect. A 582, 611 (2007).

[13] M. Honda et al., Phys. Rev. D 75, 043006 (2007).

[14] D. Casper, Nucl. Phys. B, Proc. Suppl. 112, 161 (2002).

[15] K. Eguchi et al. (KamLAND), Phys. Rev. Lett. 92, 071301 (2004).

[16] B. Aharmim et al. (SNO), Phys. Rev. C 72, 055502 (2005).

[17] J. N. Bahcall et al., Astrophys. J. 621, L85 (2005).

[18] A. Rocholl and K. P. Jochum, Earth Planet. Sci. Lett. 117, 265 (1993).

[19] J. M. Herndon, Proc. Natl. Acad. Sci. U.S.A. 100, 3047 (2003). 